\title{
Étude Raman et cristallographique de la transition de phase displacive quadratique-cubique dans une zircone nanométrique
}

\author{
P. Bouvier, V. Dmitriev ${ }^{1}$ et G. Lucazeau \\ LEPMI, CNRS INPG; 1130 rue de la Piscine, BP. 75, 38402 Saint-Martin-d'Hères cedex, \\ France \\ ${ }^{1}$ ESRF, SNBL, BP. 220, 38043 Grenoble, France
}

\begin{abstract}
RESUMÉ
L'étude par spectrométrie Raman et par diffraction de RX sous haute pression (40GPa) d'une zircone quadratique nanométrique non dopée $\left(\mathrm{P}_{2} / \mathrm{nmc} \mathrm{Z}=2\right)$ a permis de mettre en évidence une transition de phase vers une forme cubique de type fluorine ( $\mathrm{Fm} 3 \mathrm{~m} \mathrm{Z}=1$ ). La nature displacive de la transformation est discutée. En particulier, le mode mou identifié sous pression dans la phase quadratique n'étant pas le mode totalement symétrique, nous montrerons que la transition de phase fait intervenir une phase intermédiaire $\left(\mathrm{P}_{2} / \mathrm{mnm} Z=4\right)$. Un modèle phénoménologique impliquant plusieurs paramètres d'ordre est présenté sur la base du formalisme de Landau.
\end{abstract}

\section{INTRODUCTION}

La transition de phase quadratique-cubique $\left(\mathrm{D}_{4 \mathrm{~h}}{ }^{15}-\mathrm{O}_{\mathrm{h}}{ }^{5}\right)$ de la zircone réalisée thermiquement $\left(1240^{\circ} \mathrm{C}\right)$ est considérée comme étant une transition du second ordre. Les analyses théoriques montrent que dans la phase de plus grande symétrie (cubique) le mode $\mathrm{X}_{6}$ de bord de zone est instable $[1,2,3]$ et qu'il devrait posséder toutes les caractéristiques d'un mode mou observable en Raman dans la forme quadratique et capable de piloter la transition displacive cubique-quadratique. Cependant, aucune mesure expérimentale n'a permis de confirmer la présence d'un mode mou $\left(\mathrm{A}_{\mathrm{lg}}\right)$ qui puisse confirmer le caractère displacif de cette transition. La forme quadratique $D_{4 h}{ }^{15}$ peut toutefois être obtenue à pression et température ordinaires soit par dopage soit par effet de taille des cristallites [4]. L'étude présentée ici est réalisée par spectrométrie Raman et par diffraction de RX sous pression ( $40 \mathrm{GPa}$ ) et porte sur une zircone quadratique nanométrique $18 \mathrm{~nm}$ non dopée. Ce composé est dans une forme métastable dans la mesure où sa taille de cristallite doit être inférieure à $8 \mathrm{~nm}$ pour qu'elle soit dans sa forme stable à $\mathrm{T}$ et $\mathrm{P}$ ambiantes [5]. Nous montrons ici que l'effet d'une pression isostatique ne déstabilise pas l'équilibre métastable mais qu'au contraire le composé évolue vers la forme cubique de type fluorine $(\mathrm{Fm} 3 \mathrm{~m} \mathrm{Z}=1)$. L'ensemble des résultats est présenté dans la référence [6].

\section{CONDITIONS EXPÉRIMENTALES}

Les échantillons sont des poudres polycristallines préparées par procédé pyrosol décrit dans [4]. Ce procédé permet d'obtenir des tailles nanométriques (ici $18 \mathrm{~nm}$ ). La taille moyenne des cristallites a été déterminée par DRX et à partir d'observations MET. Les mesures sous pression ont été réalisées en cellule à enclume de diamant à l'ESRF sur la ligne ID30 pour la DRX et sur un spectromètre Raman XY Dilor (excitatrice $514.5 \mathrm{~nm}$ ).

\section{RÉSULTATS}

Les résultats Raman et DRX montrent que la zircone quadratique évolue à haute pression vers une phase cubique de type fluorine. Le spectre Raman évolue vers un massif unique centré vers $700 \mathrm{~cm}^{-1}$ qui pourrait correspondre à l'unique mode $T_{2 g}$ attendu dans cette structure cubique $[7,8]$. 
Sous pression, le mode à $270 \mathrm{~cm}^{-1}$ s'amollit et s'hybride avec le mode à $150 \mathrm{~cm}^{-1}$ (même intensité et même demi-largeur cf. fig. 1). Dans ce processus, les deux modes se repoussent de sorte que le mode de basse fréquence est forcé de s'amollir tandis que le mode de plus haute fréquence se stabilise à $190 \mathrm{~cm}^{-1}$. Ces observations sont typiques d'un phénomène de non-croisement [9]. Les deux modes présentent donc la même symétrie (deux modes de même symétrie ne peuvent pas adopter la même énergie et donc se repoussent). Or étant donné qu'il n'existe qu'un seul mode de symétrie $\mathrm{A}_{1 \mathrm{~g}}$ actif en Raman dans la structure quadratique et que l'interaction observée ne peut pas être attribuée à un phénomène de résonance de Fermi, dans la mesure où les deux modes présentent des intensités similaires bien avant qu'ils n'interagissent, nous devons admettre que les deux modes de basse fréquence possèdent tous deux une symétrie soit $\mathrm{B}_{\mathrm{lg}}$ soit $\mathrm{E}_{\mathrm{g}}$. Le mode mou identifié sous pression n'est donc pas le mode totalement symétrique $\mathrm{A}_{1 \mathrm{~g}}$, candidat idéal pour piloter la transition.

\section{DISCUSSION}

Dans les premières attributions de Feinberg et Perry, les deux modes basse fréquence présentent respectivement les symétries $B_{1 g}$ et $E_{g}[10]$. Mirgorodski et al. à partir de calculs de dynamique de réseau [11] ont remis en question ces attributions en proposant les symétries $A_{I g}$ et $E_{g}$. Dans les deux cas les modes sont donc autorisés à se croiser. Bouvier [6] à partir de calculs de modes normaux a montré que les deux modes qui refusent de se croiser étaient de symétrie $\mathrm{E}_{\mathrm{g}}$.

Deux possibilités peuvent donc être envisagées :

soit les deux modes ne possèdent pas la même symétrie et dans ce cas il faut admettre que la transition quadratique-cubique fait intervenir une phase intermédiaire dans laquelle les deux modes basse fréquence adoptent la même symétrie, soit les deux modes sont $E_{\mathrm{g}}$ et dans ce cas il faut expliquer comment un mode non totalement symétrique peut être un mode mou.

La topologie des zones de Brillouin des phases mère et fille montre que seul le point $X$ de bord de zone de Brillouin $\left(\mathrm{k}_{\mathrm{X}}{ }^{2}=0,0,2 \pi / \mathrm{a}\right)$ dans la phase cubique peut se condenser en centre de zone dans la phase quadratique. L'analyse de la symétrie des différents modes au point $\mathrm{X}$ permet de connâtre les différentes phases possibles. Les phases $D_{4 h}^{1}$ et $D_{4 h}{ }^{16}, D_{4 h}{ }^{5}$

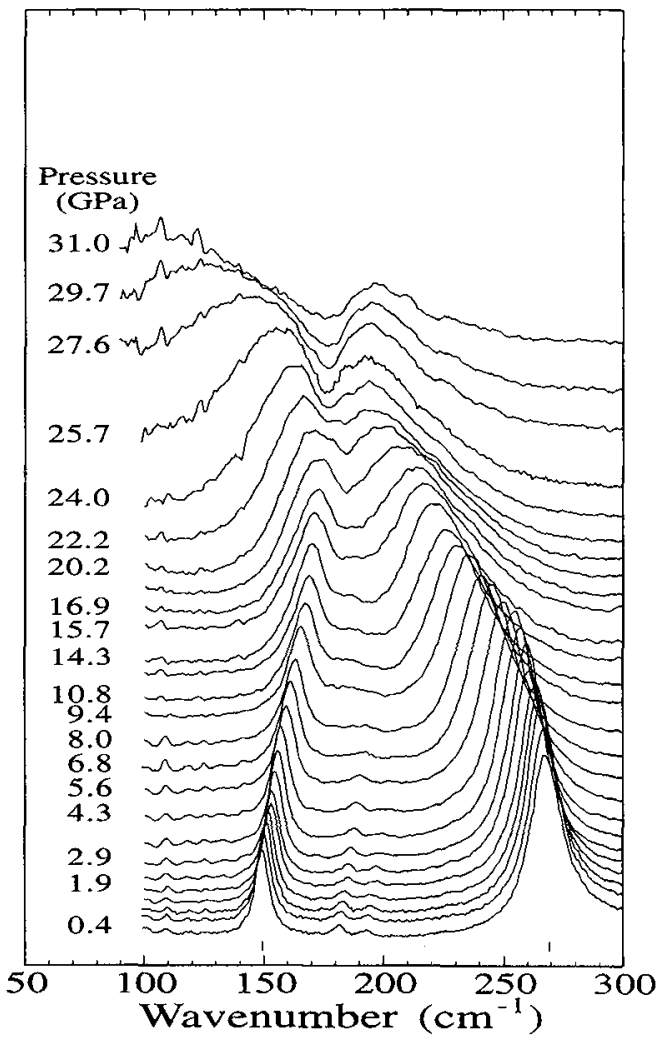

Fig I : Non-croisement de modes Raman de basse fréquence. peuvent être écartées dans la mesure où elles correspondent à des transitions du premier ordre et ne satisfont pas à la théorie de Landau. Les phases $\mathrm{D}_{4 \mathrm{~h}}{ }^{7}, \mathrm{D}_{4 \mathrm{~h}}{ }^{10}$ et $\mathrm{D}_{4 \mathrm{~h}}{ }^{3}$ peuvent être aussi écartées dans la mesure où elles ne permettent pas de reproduire la répulsion des modes basse fréquence ou imposent des répulsions entre modes qui ne sont pas observées expérimentalement. Il reste la phase $\mathrm{D}_{4 h}{ }^{14}$ qui serait issue de la condensation du mode $\mathrm{X}_{10}$. Cette phase pourrait donc être la phase intermédiaire observée entre 10 et $30 \mathrm{GPa}$. 
La figure 2 présente les filiations entre modes de centre et de bord de zone lors de la succession de transitions $\mathrm{D}_{4 \mathrm{~h}}{ }^{15}(\mathrm{I}) \rightarrow \mathrm{D}_{4 \mathrm{~h}}{ }^{14}(\mathrm{II}) \rightarrow \mathrm{O}_{\mathrm{h}}{ }^{5}$. Par exemple, l'unique mode actif $\mathrm{T}_{2 \mathrm{~g}}$ de centre de zone dans la phase cubique se décompose en deux modes $\mathrm{B}_{2 \mathrm{~g}}$ et $\mathrm{E}_{\mathrm{g}}$ de centre de zone dans la phase II. Ces modes deviennent les modes $B_{1 g}$ et $E_{g}$ de centre de zone dans la phase I. Similairement lors des transitions de phase, les modes de bord de zone de la phase cubique se condensent en centre de zone (point $\Gamma$ ) et deviennent actifs en Raman ou en infra-rouge.

Le passage par une phase intermédiaire $\mathrm{D}_{4 \mathrm{~h}}{ }^{14}$ permet d'expliquer l'ensemble des observations spectroscopiques identifiées sous haute pression (cf. figure 2 ).

(i) Les modes $\mathrm{E}_{\mathrm{g}}$ de la phase I se décomposent en 3 modes actifs en Raman lors de la transition de phase I-II. Cette augmentation du nombre de modes permet d'expliquer l'élargissement important observé pour les modes $\mathrm{E}_{\mathrm{g}}$ pour $\mathrm{P}>10 \mathrm{GPa}$. Les modes conservent des symétries identiques dans la phase II, ce qui explique le phénomène de répulsion et d'hybridation observé. Ces modes disparaissent ensuite en bord de zone lors de la transition vers la phase cubique. Il existe un mode mou totalement symétrique $\mathrm{A}_{\mathrm{lg}}$ en accord avec la théorie de Landau.

(ii) Les modes $\mathrm{E}_{\mathrm{g}}$ et $\mathrm{B}_{\mathrm{lg}}$ de la phase I se décomposent en deux modes $\mathrm{E}_{\mathrm{g}}$ et $\mathrm{B}_{2 \mathrm{~g}}$ dans la phase II puis se regroupent en un mode unique $\mathrm{T}_{2 \mathrm{~g}}$ dans la phase cubique. Ce mode est l'unique mode observé à haute pression par spectrométrie Raman.

(iii) La première transition de phase I-II impose l'apparition de deux nouveaux modes $E_{g}$ et $B_{2 g}$ qui disparaissent ensuite en bord de zone dans la phase cubique. Ces modes pourraient correspondre aux raies peu intenses observées à 300 et $700 \mathrm{~cm}^{-1}$ à haute pression.

Finalement, si la structure évolue vers une forme intermédiaire $\left(\mathrm{D}_{4 \mathrm{~h}}{ }^{14}\right)$, les attributions proposées par Mirgorodski pour la phase quadratique (mode $A_{1 \mathrm{~g}}$ à $260 \mathrm{~cm}^{-1}$ et mode $E_{\mathrm{g}}$ à $\left.150 \mathrm{~cm}^{-1}[12]\right)$ pourraient permettre d'expliquer le non croisement observé vers $15 \mathrm{GPa}$. En effet, le mode $A_{1 g}$ conserve une symétrie $A_{1 g}$ dans la phase intermédiaire tandis que le mode $\mathrm{E}_{\mathrm{g}}$ se décompose en trois modes $A_{1 \mathrm{~g}}, B_{1 \mathrm{~g}}$ et $\mathrm{E}_{\mathrm{g}}$. Il existe donc deux modes de même symétrie (ici $A_{1 g}$ ) ce qui permet de justifier le non croisement. Cependant, ces deux modes présentent un élargissement significatif vers $6 \mathrm{GPa}$ soit plusieurs $\mathrm{GPa}$ avant le non croisement. Ces élargissements ne sont donc pas uniquement caractéristiques de l'interaction (hybridation) entre ces deux modes. Ils doivent plutôt être caractéristiques de la transition vers la phase intermédiaire. Dans ce cas,

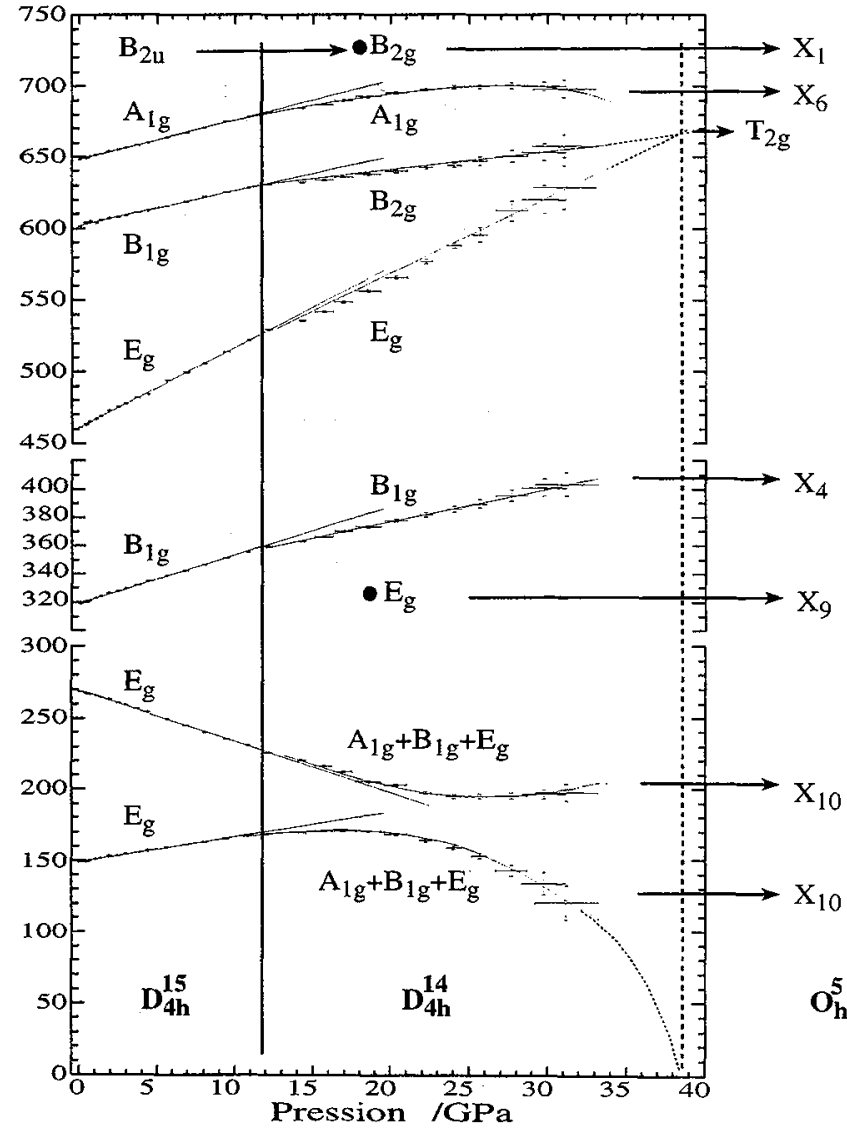

Fig 2: Filiations des modes dans la succession de transitions de phases $D_{4 h}^{15}(I)-D_{4 h}{ }^{14}(I I)-O_{h}{ }^{5}$ 
les attributions de Mirgorodski ne sont pas compatibles avec un élargissement simultané des deux modes.

\section{Apport de la théorie de Landau des transitions de phases}

Dans le formalisme de Landau, cette succession de transitions de phases peut être décrite par un potentiel de Landau $F$ faisant intervenir deux invariants $I_{l}$ et $I_{2}$.

$$
\begin{aligned}
F & =\frac{1}{2} a_{1} I_{1}^{2}+\frac{1}{4} a_{2} I_{1}^{4}+\frac{1}{6} a_{3} I_{1}^{6}+\cdots \\
& +\frac{1}{2} b_{1} I_{2}^{2}+\frac{1}{4} b_{2} I_{2}^{4}+\cdots+\delta I_{1}^{2} I_{2}^{2}
\end{aligned}
$$

avec $I_{1}^{2}=\eta_{1}{ }^{2}+\eta_{2}{ }^{2}+\eta_{3}{ }^{2}$ décrit par le phonon $\mathrm{X}_{6}$ et $I_{2}{ }^{2}=\varsigma_{1}{ }^{2}+\ldots+\zeta_{6}{ }^{2}$ décrit par le phonon $\mathrm{X}_{10}$. Pour des raisons de symétrie, le potentiel de Landau fait donc intervenir un couplage bi-quadratique entre ces deux invariants. Les différentes phases produites sont décrites par Tolédano et Dmitriev en fonction des puissances introduites dans le potentiel de Landau [13]. En substance, la topologie des frontières d'équilibre est directement dépendante du coefficient de couplage $\delta$.

Dans le cas particulier où les transitions O-I et O-II sont des transitions du second ordre (c'est-à-dire que $a_{2}{ }^{2}>0, b_{2}{ }^{2}>0$ et $\Delta=4 a_{2}{ }^{2} b_{2}{ }^{2}-\delta^{2}<0$ ), le diagramme de phase correspondant est représenté dans le plan paramètrique $\left(a_{1}, b_{1}\right)$ à la figure 3 . Les coefficients $a_{1}$ et $b_{1}$ sont supposés varier linéairement avec la Température et/ou la Pression.

La mesure des variations de fréquence des phonons $\mathrm{X}_{6}$ et $\mathrm{X}_{10}$ sous l'influence de la pression et de la température, voire de la déformation de la maille (qui pourrait être introduite comme un paramètre secondaire dans le développement de Landau ), pourrait permettre de déterminer les coefficients $a_{i}$ et $b_{i}$. La figure présente les axes $P$ et $T$ supposés représenter correctement les transitions de phase observées. Ce diagramme permet en outre d'apporter une vision unifiée des différents travaux de la littérature. L'effet seul de la température conduit à traverser la frontière I-O tandis que l'effet de pression conduit à traverser les frontières I-II-O.

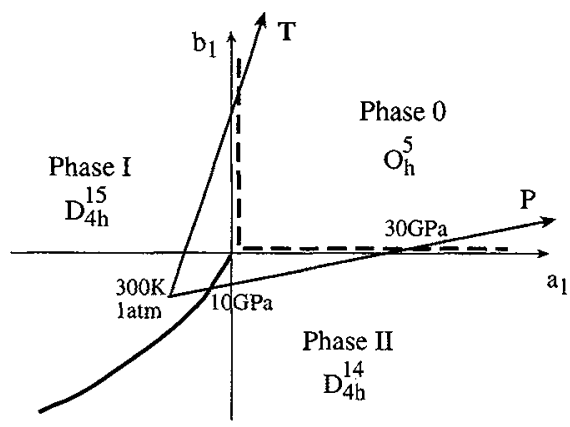

Fig 3: lignes d'équilibre entre phases dans le plan paramétrique $\left(a_{l}, b_{l}\right)$ : Trait continu $=$ transition $d u I^{e r}$ ordre, traits en pointille = transitions $d u$ 2nd ordre

\section{CONCLUSION}

La forme quadratique $\mathrm{P} 4_{2} / \mathrm{nmc}$, métastable n'est pas déstabilisée par l'application d'une pression isostatique et se transforme vers une forme cubique fluorine idéale (sans lacunes). Les deux modes Raman de basse fréquence qui dans un premier temps se rapprochent sous l'effet de la pression appartiennent à la symétrie Eg dans la phase quadratique initiale (1 atm-12GPa). Ils donnent naissance à des multiplets, source d'élargissement des bandes Raman dans la phase quadratique 
intermédiaire $\mathrm{P}_{2} / \mathrm{mnm} \mathrm{Z}=4(12-38 \mathrm{GPa})$ et la composante $\mathrm{A}_{\mathrm{Ig}}$ peut piloter la transition vers la forme cubique.

\section{REFÉRENCES}

[1] A.P. Mirgorodsky, M.B. Smirnov, P.E. Quintard, T. Merle-Méjean, Phys. Rev. B 52 (1995) 9111.

[2] A.P. Mirgorodsky, M.B. Smirnov and P.E. Quintard, Phys. Rev. B 55 (1997) 19.

[3] J.K. Dewhurst and J.E. Lowther, Phys. Rev. B 57 (1998) 741.

[4] Djurado E., Bouvier P. and Lucazeau G., J. Solid State Chem. 149, 399 (2000)

[5] P Bouvier, J. Godlewski, G. lucazeau, XXVII JEEP et J Nuclear Mat (soumis)

[6] P. Bouvier Thèse INPG 2001

[7]P.Bouvier and G.Lucazeau, J. Phys. Chem. Solids 61 (2000) 569.

[8] P.Bouvier, E.Djurado, G.Lucazeau and T. Le Bihan, Phys. Rev. B 62 (2000) 8731.

[9] V.L. Ginzburg, A.A. Sobyanin, A.P. Levanyuk, General Theory of light scattering near phase transitions in ideal crystals, modern problems in condensed matter science, Vol5, NH 1983

[10] Feinberg and Perry, J.Phys.Chem Solids 42, (1981)513

[11] A.P.Mirgorodsky, M.B.Smirnov and P.E.Quintard, J. Phys. Chem. Solids 60 (1999) 985.

[12] A. Feinberg and C.H. Perry, J. Phys. Chem. Solids 42 (1981) 513.

[13] P.Tolédano and V.Dmitriev, reconstructive phase transition in crystals and quasicrystals, World Scientific, Singapore (1996) 